\title{
Energiedienstleistungen zur Unterstützung von Endverbrauchern
}

\author{
Um die Klimaschutzziele zu erreichen, muss \\ beim Wohnen viel Energie eingespart werden. \\ Doch wie viel Energie kann man durch Verhaltens- \\ änderungen einsparen? Ein EU-Projekt bietet mit \\ Energiedienstleistungen Unterstützung für \\ Endverbraucher. \\ Von Michael Scharp
}

$\mathbf{F}_{2}^{\mathrm{u}}$ ür das Wohnen wurden in Deutschland im Jahre 2009 etwa 29 Prozent der gesamten Endenergie genutzt (BMWi 2010). Trotz intensiver Sanierung des Wohnungsbestandes lag der durchschnittliche Energieverbrauch für Heizung und Warmwasser in 2007 noch bei etwa $140 \mathrm{~kW} / \mathrm{m}^{2}$ Wohnfläche. Der Stromverbrauch pro Person betrug im selben Jahr etwa 940 kWh (ohne Strom für Heizzwecke), sodass jeder Bundesbürger im Durchschnitt etwa $7.800 \mathrm{kWh}$ für das Wohnen verbrauchte (eigene Berechnung nach BMWi 2010). Angesichts einer inzwischen weitgehenden Einigkeit in Politik und Gesellschaft, dass wir immer noch nicht genug Energie eingespart haben, um wenigsten das zwei-Grad-Ziel zu erreichen, stellt sich die Frage, wie man Haushalte unterstützen kann, noch mehr Energie als bisher einzusparen.

Vor diesem Hintergrund wurde das BewareE-Projekt zwischen 2007 bis 2010 von vier Forschungsinstituten in Deutschland, den Niederlanden und Spanien durchgeführt. BewareE wurde von der EACI European Agency of Competitiveness and Innovation gefördert. Das Forschungskonzept sah vor, eine europaweite Identifikation und Analyse von Energiedienstleistungen durchzuführen, gute Beispiele auszuwählen und diese durch Vorträge und Veröffentlichungen zu verbreiten. Dieses einfache Konzept musste immer wieder geändert werden, da sich im Laufe der Zeit interessante und wichtige Fragestellungen ergaben.

\section{Was sind Energiedienstleistungen?}

Der Begriff Energiedienstleistungen ist inzwischen weit verbreitet. Die VDI-Richtlinie von 2001 beschrieb ihn wie folgt: „Energiedienstleistungen sind die aus dem Einsatz von Nutzenergie und anderen Produktionsfaktoren befriedigten Bedürfnisse bzw. erzeugten Güter.“ Unter Bedürfnisse werden beispielsweise beheizte oder beleuchtete Räume verstanden. Diese
Definition ist jedoch nur bedingt tragfähig, da anerkannte Dienstleistungen wie eine „Energieberatung“ kaum unter diese Vorstellung von Bedürfnissen fallen.

Die Energiedienstleistungsrichtlinie der EU von 2006 erwies sich nur teilweise als hilfreich. Nach der Definition der EU-Richtlinie ist eine „Energiedienstleistung [ist] der physikalische Nutzeffekt, der Nutzwert oder die Vorteile als Ergebnis der Kombination von Energie mit energieeffizienter Technologie und / oder mit Maßnahmen, die die erforderlichen Betriebs-, Instandhaltungs- und Kontrollaktivitäten zur Erbringung der Dienstleistung beinhalten können; sie wird auf der Grundlage eines Vertrags erbracht und führt unter normalen Umständen erwiesenermaßen zu überprüfbaren und mess- oder schätzbaren Energieeffizienzverbesserungen und / oder Primärenergieeinsparungen. “ Zum einen ist die Definition sehr sperrig, zum anderen schließt sie zahlreiche im allgemeinen Sprachgebrauch übliche und anerkannte Energiedienstleistungen aus. Die Energieberatung kann beispielsweise nicht garantieren, dass der Dienstleistungsnehmer auch tatsächlich Energie einspart.

Selbst wenn durch spätere Erhebungen in Erfahrung gebracht wird, dass xy Haushalte Energieeffizienzinvestitionen getätigt haben, kann nicht kausal von der Beratung auf das Investment geschlossen werden. Ebenso fallen alle Beratungsleistungen, die nicht auf Basis eines Vertrages erbracht werden, unter diese Definition. Das BewareE-Team musste deshalb mit einer neuen Definition sich eine eigene Arbeitsgrundlage schaffen. Deshalb verstehen wir unter Energiedienstleistungen für Haushalte: Eine Energiedienstleistung ist jede Art von Handlung oder Maßnahme, die auf Wohnungsnutzer zielt und diese dabei unterstützt, im Umgang mit Energie eine nachhaltigere Verhaltensweise zu erlangen.

\section{Arten und Verbreitung}

Energiedienstleistungen und die eingesetzten Mittel können deshalb vielfältig sein. Sie können Kampagnen, Informationsmaterialien, Veranstaltungen, Ausstellungen, Webseiten, Trainingsprogramme, Analysen und Beratungen zu Energiethemen umfassen. Das Ziel der Dienstleistung soll die Energieeinsparung und / oder die effizientere Nutzung von Energie im Haushalt sein. Die Verhaltensänderungen können sich auf das Konsum- und Investitionsverhalten sowie den alltäglichen Umgang mit Energie beziehen. Für das BewareE-Projekt waren nur solche Dienstleistungen relevant, die für die Haushalte nicht mit großen Kosten (Investitionen) verbunden waren. Die Zielgruppe der Dienstleistungen waren Mieter, Nutzer und Eigentü- $\rightarrow$ 
mer von Wohnungen, die Anbieter konnten Wohnungsunternehmen oder andere Institutionen sein.

Mit dieser Definition im Hintergrund wurde eine umfangreiche Recherche nach Dienstleistungen in allen Ländern der Europäischen Union (EU) durchgeführt. Wie nicht anders zu erwarten zeigten sich deutliche Unterschiede im Umfang und in der Ausgestaltung des Dienstleistungsangebotes. In den nord- und mitteleuropäischen Ländern sowie in Großbritannien gab es eine Fülle von Dienstleistungen unterschiedlicher Ausprägungen. In den süd- und osteuropäischen Ländern hingegen konnten trotz intensiver Recherche nur wenige Dienstleistungsangebote ausfindig gemacht werden. Vielfach war für dieses Angebot charakteristisch, dass die Dienstleistung im Rahmen von EU-Projekten entwickelt worden war oder dass es sich um einfache Dienstleistungen wie Web-Informationen oder Veranstaltungen handelte.

\section{Wie viel Energie kann eingespart werden?}

Nachdem etwa 140 mögliche Dienstleistungen identifiziert wurden, bestand die nächste Aufgabe in der Analyse derselben und die Auswahl von sogenannten Good Practice Beispielen. Hierbei zeigte sich sehr schnell, dass zwar viele Energiedienstleistungen angeboten werden, aber die Anbieter nur selten über Daten verfügen, um die Wirksamkeit der Dienstleistungen beurteilen zu können. Das BewareE-Team musste deshalb eine umfangreiche Literaturanalyse durchführen, um überhaupt einschätzen zu können, wie viel Energie mit welchen Dienstleistungen bestenfalls eingespart werden kann.

Interessanterweise gibt es nur wenige Studien, die sich dieses Themas angenommen haben. Hinsichtlich des gesamten Einsparpotentials durch Verhaltensänderungen gibt es nur Studien von Gardner und Stern sowie von Kok et al (Gardner/Stern 2009; Kok et al. 2007). Nach Kok kann durch ein verändertes Verhalten - dies umfasst ein energiebewusstes Verhalten, ein energieärmerer Lebensstil, mehr Aufmerksamkeit für den Energieverbrauch und geringe investive Maßnahmen - schätzungsweise fünf Prozent bis 19 Prozent unseres Energieverbrauchs eingespart werden. Gardner und Stern haben eine sogenannte Short-List mit Einsparpotentialen für die USA auf Basis der Aus- wertung von Studien erstellt. Mit nur vier Maßnahmen im Haushalt - Energiesparen beim Wäschewaschen, Abspülen, Temperaturänderungen beim Heizen und Kühlen - lassen sich fast fünf Prozent der Energie im Haushalt sparen. Nimmt man die Verhaltensänderungen in Verbindung mit geringfügigen Investitionen (beispielsweise etwas höhere Kosten für energieeffiziente Haushaltsgeräte) hinzu, kann man noch ein weiteres Potential von zwölf Prozent erschließen. Die folgende Tabelle stellt die Short-List dar:

Damit war zumindest ein Schätzwert gegeben, der die Richtung für mögliche Energieeinsparpotenziale aufzeigt. Als Nächstes betrachteten wir, welche der vielen möglichen Dienstleistungen die Haushalte dabei unterstützen kann, diese Potentiale auch zu erschließen?

Schon in den siebziger Jahren wurden die ersten Untersuchungen über die Möglichkeiten zur Förderung der Energieeffizienz bei Haushalten durchgeführt. In vielen Studien wurden die Hemmnisse und Erfolgsfaktoren zur Verhaltensänderung sowie mögliche Anreize zur Förderung des Energiesparens untersucht. Allerdings gibt es nur eine sehr beschränkte Anzahl von Studien, die auch quantitativ evaluiert hat, in welchem Umfange die betrachteten Maßnahmen das Energiesparen fördern. Abrahamse et. al. haben im Jahre 2005 in einer exzellenten Metastudie ausführlich untersucht, welche Energiedienstleistungen tatsächlich das Energiesparen fördern (Abrahamse et. al. 2005). Abrahamse unterscheidet fünf strategische Ansätze bzw. Dienstleistungstypen:

- Information: Hierunter versteht Abrahamse Kampagnen, allgemeine schriftliche Informationen, Webseiten, Workshops, Ausstellungen, Beratungen, Hausbesuche und Anderes, sofern die Aktion nur einmalig ist. Der Vorteil dieser Informationsmaßnahmen ist, dass sie zumeist preiswert sind (mit Ausnahme der persönlichen Beratungen). Es gibt jedoch keine Studie, die die Wirksamkeit der Informationsstrategie nachgewiesen hat. Nur in Verbindung mit anderen Instrumenten zeigten sich Energieeinsparungen.

- Modellierung und Vorbilder: Bei dieser Strategie rufen öffentlich bekannte Vorbilder wie zum Beispiel der Umweltminister zum Energiesparen auf. Sie wird meist mit Hilfe von Printmedien, Fernsehen und Rundfunk umgesetzt. Nur eine einzige von wenigen Studien hat gezeigt, dass eine Stromeinsparung von zehn Prozent zumindest während der Laufzeit der Kampagne erreicht wurde (Winett et al. 1985).

- Verpflichtungen und Zielsetzungen: Bei der Verpflichtungsstrategie verpflichten sich Haushalte zum Energiesparen, bei der Zielsetzungsstrategie setzen sich die Haushalte Ziele. Die Wirksamkeit von Verpflichtungen und Zielsetzungen mit Energieeinsparungen von bis zu 15 Prozent konnten nur in Verbindung mit anderen Strategien wie zum Beispiel Feed- 
back nachgewiesen werden (Pallak/Cummings 1976; Katzev/Johnson 1983; Becker 1978).

I Feedback: Die Feedback-Strategie ist eine Beratung von Haushalten über Energieverbrauch und Energienutzung. Der Vorteil ist, dass es eine sehr wirksame Methode ist, die am besten mit einem regelmäßigen Energiecontrolling zusammen funktioniert. Der Nachteil ist, dass die individuelle Beratung nur mit einem hohen Personaleinsatz durchgeführt werden kann. In verschiedenen Projekten konnten Energieeinsparungen von vier bis zwölf Prozent Strom (ohne Zielwerte) beziehungsweise bis 22 Prozent Strom (mit Zielwerten) erreicht werden (Seligman/Darby 1977; Mc Calley/Midden 2002; Brandon/Lewis 1999). McMakin zeigte in einer Studie mit etwa 1.200 Haushalten, dass diese durchschnittlich zehn Prozent Strom- und Gas einsparten (McMakin 2002).

- Anreize: Bei dieser Strategie erhalten Verbraucher Anreize wie zum Beispiel günstige Tarife, Bonuszahlungen oder günstigere Kredite, wenn sie weniger Energie verbrauchen. Der Vorteil der Strategie ist, dass es eine sehr wirksame Methode zur Verhaltensänderung ist und dass es auf die Höhe des Anreizes nicht so sehr ankommt. Der Nachteil ist, dass nach Einstellen der Anreize die Haushalte wieder mehr Energie verbrauchen. Die Wirksamkeit ist in zahlreichen Studien belegt, wobei sich zeigte, dass bis zu sieben Prozent Strom oder Gas Einsparung zu erzielen sind (Winett et al. 1985; McClelland/Cook 1980).

Zusammenfassend kann man feststellen, dass es bisher nicht ausreichend gelungen ist nachzuweisen, dass die Informationsstrategie (Flyer, Broschüren, Ausstellungen, Webseiten, Veranstaltungen, einmalige Beratungen oder Kampagnen) wirklich Haushalte motivieren, Energie einzusparen. Auch die Modellierungs-, Verpflichtungs- und Zielsetzungsstrategie ist hinsichtlich ihrer Wirksamkeit nur unzureichend untersucht worden. Allein Feedback mit individueller Beratung hingegen lehrt Haushalte das Energiesparen und Anreize fördern definitiv das Energiesparen.

Die Studien haben zudem gezeigt, dass bis 20 Prozent Energie mit einem angemessenen Methodenmix eingespart werden können. Da es sich bei den wissenschaftlichen Untersuchungen jedoch zumeist um Modellvorhaben mit intensiver wissenschaftlicher Begleitung handelte, sollte eine Initiative, die zum Energiesparen auffordert und Projekte durchführt, auch mit geringeren Einsparpotenzialen zufrieden sein. Wenn es also gelingt, zehn Prozent des Energieverbrauchs für den Haushaltssektor dauerhaft einzusparen, so ist dies bereits ein sehr großer Beitrag.

Vor diesem Hintergrund wurde die Analyse der identifizierten Energiedienstleistungen mithilfe einer vereinfachten SWOTAnalyse durchgeführt. Insgesamt wurden 38 Energiedienstleistungen als gute Beispiele ausgewählt, von denen einige im Folgenden vorgestellt werden.

Das Wohnungsunternehmen Volkswohnung GmbH Karlsruhe hat im Rahmen einer Bestandssanierung ein Modellpro- jekt zum Energie-Monitoring durchgeführt. In einem Gebäude wurde die Hälfte der Wohnungen mit elektronischen Thermostaten, Messgeräten zur Erfassung des Energieverbrauchs und des Luftwechsels sowie Energiedisplays zur Anzeige des Energieverbrauchs ausgestattet. Die erfassten Daten wurden zentral erfasst, ausgewertet und für die Display-Darstellung aufbereitet. Der tägliche Energieverbrauch wurde auf dem Display durch drei Typen von Smileys angezeigt. Im Vergleich zu den anderen Wohnungen im gleichen Gebäude, die nicht mit Messgeräten und dem Display ausgestattet wurden, verbrauchten die Mieter mit den Displays sieben Prozent weniger Energie.

\section{Schulung einkommensschwacher Haushalte}

Das Projekt “Energie op Maat” wurde von der Kommune Dordrecht entwickelt, um Haushalte mit geringem Einkommen in ihrer Energienutzung zu beraten. Hierzu besuchen Energieberater die teilnehmenden Haushalte vor Ort, analysieren zusammen mit den Bewohnern den Energiegebrauch und machen anschließend individuelle Vorschläge zum Energiesparen. Die Energieberater verteilen dabei kostengünstige Hilfsmittel wie Energiesparlampen, Perlatoren oder Dichtungsstreifen für Fenster. Durchschnittlich konnten bei den teilnehmenden Haushalten etwa zwölf Prozent an Gas und Elektrizität eingespart werden.

Bei dem komparativen Feedback erhalten Haushalte Informationen, die einen Vergleich mit anderen Energieverbrauchern ermöglichen. Der schwedische Energieversorger „Umeå Energi“ hat Ende der 90iger-Jahre einen Energiebrief für Haushalte entwickelt. Die Haushalte übersandten dem Energieversorger ihre Verbrauchswerte und einige Daten zu den Wohngebäuden. Der Energieversorger bereinigte den Verbrauch um Witterungseinflüsse, fügte vergleichbare Verbrauchswerte anderer Haushalte hinzu und gab Vorschläge zur Minderung des Verbrauchs. In einer Pilotstudie wurde gezeigt, dass die beteiligten Haushalte ihren Energieverbrauch um 2.400 kWh - zwölf Prozent des Jahresverbrauchs - reduziert hatten.

\section{Bewohner trainieren Bewohner}

Im Rahmen des finnischen „Energy Expert Training”-Projekts werden Mieter des finnischen Wohnungsunternehmens VVO von der nationalen Energieagentur Motiva Oy zu Energieexperten für die Mieterberatung geschult. Ihre wesentliche Aufgabe ist die Beratung ihrer Mitmieter zu Fragen der Energieeffizienz und -einsparung. Weiterhin kontrollieren sie den gebäudebezogenen Energie- und Wasserbedarf des Gebäudes (Treppenhausbeleuchtung, Heizungsstrom, Grünanlagenbewässerung). Die VVO verfügte in 2007 innerhalb ihrer Bewohnerschaft über 500 Experten. Der Verbrauch in den Häusern mit den Energieexperten konnte im Vergleich zu Liegenschaften ohne Experten deutlich gesenkt werden: Es wurden fünf Prozent weniger Heizenergie, zehn Prozent weniger Elektrizität und 20 Prozent weniger Wasser verbraucht. 
Einige belgische Kyoto-Vereine, die das gemeinsame Ziel haben die Kohlentsoffdioxid-Emission um zehn Prozent zu senken, haben die Kampagne „Knack den Kyoto Code“ für Haushalte initiiert. Die Haushalte erhielten ein Direktivenbuch mit Energiespartipps und eine Anleitung zur Energieanalyse. Über einen Zeitraum von drei Monaten erfassten die Haushalte ihren Energieverbrauch und notierten die Häufigkeit der befolgten Ratschläge. Veranstaltungen sollten die Teilnehmer motivieren. Die Kyoto-Vereine berechneten hieraus die eingesparte Energie. Pro Haushalt waren dies 225 Kilogramm Kohlentsoffdioxid beziehungsweise 280 kWh Strom oder 1.200 kWh Gas in drei Monaten.

Im Rahmen eines Neubauprojekts der schwedischen Gemeinde Växjö wurde ein Bedarfsmanagement erprobt. Die 91 WE wurden als Niedrigenergiehäuser errichtet ( 95 beziehungsweise $85 \mathrm{kWh}$ pro $\mathrm{m}^{2} / \mathrm{a}$ ) und lagen deutlich unter dem schwedischen Durchschnitt von 140 kWh. Jede Wohnung hatte ein Messsystem für Strom, Warmwasser und Heizwärme, in einem Teil der Wohnungen waren Displays zur Darstellung des Energieverbrauchs installiert. Um die Wirksamkeit zu testen, wurden drei Nutzergruppen gebildet. Eine Gruppe hatte keine Informationen und Displays, die anderen Gruppen mit Displays erhielten keine beziehungsweise verbrauchsorientierte Informationen. Das Display führte zu einer Reduktion des Stromverbrauchs um 24 Prozent, mit den zusätzlichen Informationen sank der Verbrauch sogar um 34 Prozent. Der Warmwasserverbrauch sank gegenüber den Display-Gruppen um 43 Prozent, das heißt, die zusätzlichen Informationen hatten hier keinen Effekt.

\section{Technische Hilfsmittel zum Stromsparen}

Die dänische Energieagentur „Energi“ hat ein Forschungsprojekt zur Minderung des Stand-by Verbrauchs elektrischer Geräte, der in Dänemark auf durchschnittlich acht Prozent beziffert wird, durchgeführt. In dem Projekt wurden unterschiedliche Hilfsmittel verwendet wie zum Beispiel schriftliche Informationen, eine Webseite mit der Darstellung des Stromverbrauchs der Haushalte, persönliche Energieberatungen (Feedback) oder technische Hilfsmittel wie Steckerleisten und Anzeigegeräte. Die schriftlichen Informationen zeigten keinen Effekt, die Energieberatung reduzierte den Stand-by Verlust um etwa 40 Prozent und bei gleichzeitiger Nutzung von technischen Hilfsmitteln reduzierte sich der Verlust um etwa 65 Prozent.

\section{Anmerkungen}

Weitergehende Informationen:

Scharp, Michael (2010): BewareE Handbuch: Energiedienstleistungen für Mieter und die Wohnungswirtschaft. IZT WerkstattBericht Nr. 110. Berlin: IZT Institut für Zukunftsstudien und Technologiebewertung. (download unter http://www.izt.de).

Scharp, Michael (2010): BewareE Manual: Energiedienstleistungen für Haushalte. IZT WerkstattBericht Nr. 108. Berlin: IZT Institut für Zukunftsstudien und Technologiebewertung. (download unter http://www.izt.de.)

\section{Literatur}

Abrahamse, W. et al.: Review of intervention studies aimed at household energy conservation. In: Journal of Environmental Psychology 25/2005, S. 273-91.

Becker, L.J.: Joint effect of feedback and goal setting on performance: A field study of residential energy conservation. In: Journal of Applied Psychology 63/1978, S. 428-33.

BMWi Bundesministerium für Wirtschaft und Technologie: Energiedaten Nationale und internationale Entwicklung. BMWI: Berlin 2009. Online: www.bmwi.de / BMWi / Navigation / Energie / energiestatistiken.html. Zugriff: Januar 2011

Brandon, G. / Lewis, A.: Reducing household energy consumption: A qualitative and quantitative field study. In: Journal of Environmental Psychology 19/1999, S. 75-85.

Europäische Kommission: Richtlinie 2006 / 32 / EG über Endenergieeffizienz und Energiedienstleistungen. Brüssel 2006. Online: eur-lex.europa.eu / LexUriServ / LexUriServ.do?uri=OJ:L:2006:114:0064:0085:DE:PDF. Zugriff: Oktober 2009.

Gardner, Gerald T. / Stern, Paul C.: The Short List - The Most Effective Actions U.S. Households Can Take to Curb Climate Change. Environment - Science and Policy for Sustainable Development: Philadelphia 2009. Online: http://www.environmentmagazine.org/Archives/Back\%20lssues/September-October\%202008/gardner-stern-full.html. Zugriff: Januar 2010.

Katzev, R. / Johnson, T.R.: A social-psychological analysis of residential electricity consumption: The impact of minimal justification techniques. In: Journal of Economic Psychology 3/1983, S. 267-84.

McCalley, L.T. / Midden, C.J.H.: Energy conservation through product-integrated feedback. In: Journal of Economic Psychology 23/2002, S. 589-603

McClelland, L. / Cook, S.W.: Energy conservation effects of continuous inhome feedback in all-electric homes. In: Journal of Environmental Systems 9/1979-80, S. 169-73.

McMakin, A.H. et al.: Motivating residents to converse energy without financial incentives. In: Environment and Behavior 34/2002, S. 848-63.

Pallak, M.S. / Cummings, N.: Commitment and voluntary energy conservation. In: Personality and Social Psychology Bulletin 2/1976, S. 27-31

Seligman, C. / Darby, J.M.: Feedback as means of decreasing residential energy consumption. In: Journal of Applied Psychology 62/1977, S. 363-8.

VdI: VDI Richtlinie 4661. Zitiert nach Enquete-Kommission - Nachhaltige Energieversorgung, 2001. Online: webarchiv.bundestag.de / archive / 2007 / 0108 / parlament / gremien / kommissionen / archiv14 / ener / schlussbericht / 10_4AnhangRaster.pdf. Zugriff Januar 2008.

Kok, G. / Jonkers, R. / Uitdenbogerd, D.: Energieverbruik en gedragsverandering in de woning. Een literatuuronderzoek naar determinanten en interventieprogramma's. The Hague 2007.

Winett, R.A. et al. : Effects of television modeling on residential energy conservation. In: Journal of Applied Behavior Analysis 18/1985, S. 33-44.

\section{IAUTOR + KONTAKT}

Dr. Michael Scharp ist seit 1995

Wissenschaftler am IZT Institut für Zukunftsstudien und Technologiebewertung mit Arbeitsschwerpunkten in den Bereichen Instrumente zur Innovationsförderung, nachhaltiges Bauen und Wohnen sowie Dienstleistungsforschung.

Dr. Michael Scharp, IZT Institut für Zukunftsstudien und Technologiebewertung, Schopenhauerstraße 26,

14129 Berlin. Tel.: +4930 803088-14, Fax: +4930803088-88, E-Mail: m.scharp@izt.de 


\section{Lizenzhinweis}

Die Beiträge in ÖkologischesWirtschaften werden unter der Creative-Commons-Lizenz "CC 4.0 Attribution Non-Commercial No Derivatives" veröffentlicht. Im Rahmen dieser Lizenz muss der Autor/Urheber stets genannt werden, das Werk darf nicht bearbeitet, abgewandelt oder in anderer Weise verändert und außerdem nicht kommerziell genutzt werden. Die digitale Version des Artikels bleibt für zwei Jahre Abonnent/innen vorbehalten und ist danach im Open Access verfügbar. 